\title{
Plasma-derived Exosomes Reverse Epithelial-to-Mesenchymal Transition after Photodynamic Therapy of Patients with Head and Neck Cancer
}

\author{
Marie-Nicole Theodoraki ${ }^{1,2 *}$, Saigopalakrishna S. Yerneni ${ }^{3 *}$, Cornelia Brunner ${ }^{2}$, \\ Joannis Theodorakis ${ }^{4}$, Thomas K. Hoffmann ${ }^{2}$ and Theresa L. Whiteside ${ }^{1,5}$ \\ ${ }^{1}$ Department of Pathology, University of Pittsburgh School of Medicine and UPMC Hillman Cancer Center, Pittsburgh, PA \\ 15213, USA \\ 2 Department of Otorhinolaryngology, Head and Neck Surgery, University of Ulm, Germany \\ ${ }^{3}$ Department of Biomedical Engineering, College of Engineering, Carnegie Mellon University, Pittsburgh, PA 15217, USA \\ ${ }^{4}$ Department of Urology, Geniki Kliniki, Thessaloniki, Greece \\ ${ }^{5}$ Departments of Immunology and Otolaryngology, University of Pittsburgh School of Medicine, Pittsburgh, PA 15213, USA \\ * These authors contributed equally to the manuscript
}

Correspondence to: Theresa L. Whiteside, email: whitesidetl@upmc.edu

Keywords: plasma-derived exosomes; exosome-mediated reprogramming; epithelial-to-mesenchymal transition; head and neck cancer; photodynamic therapy

Received: April 06, $2018 \quad$ Accepted: April 21, $2018 \quad$ Published: April 29, 2018

Copyright: Theodoraki et al. This is an open-access article distributed under the terms of the Creative Commons Attribution License 3.0 (CCBY 3.0), which permits unrestricted use, distribution, and reproduction in any medium, provided the original author and source are credited

\section{ABSTRACT}

Photodynamic therapy (PDT) is a palliative treatment option for head and neck squamous cell carcinoma (HNSCC) patients which induces local inflammation and alters tumor cell morphology. We show that exosomes in plasma of HNSCC patients undergoing PDT reprogram tumor cells towards an epithelial phenotype. Nine HNSCC patients were treated with PDT and plasma was collected prior to and at three timepoints after therapy. Exosome levels of E-Cadherin, $\mathrm{N}$-Cadherin and TGF- $\boldsymbol{\beta} 1$ were tested by flow cytometry. Exosomes were co-incubated with cancer cells, and changes in expression of EMT markers were evaluated as were proliferation, migration, chemotaxis and invasiveness of tumor cells. Exosomes harvested preand 24h after PDT were enriched in N-Cadherin and TGF- $\beta 1$. They induced the mesenchymal phenotype and up-regulated Vimentin and transcripts for Snail, Twist, a-SMA, Slug and ZEB1 in epithelial tumor cells. These exosomes also enhanced tumor proliferation, migration and invasion. In contrast, exosomes obtained on day 7 or 4-6 weeks after PDT carried E-cadherin, restored epithelial morphology and EPCAM expression in tumor cells, down-regulated expression of mesenchymal genes and inhibited proliferation, migration and invasion. The PDT-mediated conversion from the mesenchymal to epithelial tumor phenotype was mediated by exosomes, which also served as non-invasive biomarkers of this transition.

\section{INTRODUCTION}

Despite the currently available treatment options for patients with advanced Head and Neck Squamous Cell Carcinoma (HNSCC), the disease outcome remains poor, mostly due to locoregional tumor recurrence. Therapy options are limited for patients with recurrent HNSCC. The standard of care is palliative chemotherapy [1]. Today,
PD-1 inhibitors represent a newly approved therapeutic option for these patients $[2,3]$. An alternative treatment option for selected cases of recurrent and therapyrefractive HNSCC is the photodynamic therapy (PDT) with Temoporfin (Foscan ${ }^{\circledR}$, Biolitec, Austria) [4-7]. Effects of PDT on the tumor may vary. PDT might induce tumor necrosis and tissue damage, leading to local inflammation, infiltration of immune cells and activation of long-term 
Table 1: Clinicopathological data

\begin{tabular}{|c|c|c|}
\hline & \multicolumn{2}{|c|}{$\begin{array}{c}\text { Patients } \\
\quad(n=9)\end{array}$} \\
\hline & $\mathrm{n}$ & $\%$ \\
\hline \multicolumn{3}{|l|}{ Age (years) } \\
\hline$\leq 60$ & 4 & 44 \\
\hline$>60$ & 5 & 56 \\
\hline \multicolumn{3}{|c|}{ (range: 52-79) } \\
\hline \multicolumn{3}{|l|}{ Gender } \\
\hline male & 6 & 67 \\
\hline female & 3 & 33 \\
\hline \multicolumn{3}{|c|}{ Tumor stage } \\
\hline pT1 & 3 & 33 \\
\hline pT2 & 0 & 0 \\
\hline pT3 & 1 & 11 \\
\hline pT4 & 5 & 56 \\
\hline \multicolumn{3}{|c|}{ Nodal status } \\
\hline N0 & 3 & 33 \\
\hline $\mathrm{N} \geq 1$ & 6 & 67 \\
\hline \multicolumn{3}{|c|}{ Distant metastasis } \\
\hline M0 & 8 & 89 \\
\hline M1 & 1 & 11 \\
\hline \multicolumn{3}{|c|}{ Amount of treatment lines (pre-PDT) } \\
\hline 1 & 1 & 11 \\
\hline 2 & 4 & 45 \\
\hline 3 & 2 & 22 \\
\hline 4 & 2 & 22 \\
\hline \multicolumn{3}{|c|}{ Radiotherapy } \\
\hline 0 & 1 & 11 \\
\hline 1 & 5 & 56 \\
\hline 2 & 3 & 33 \\
\hline \multicolumn{3}{|c|}{ Chemotherapy } \\
\hline 0 & 4 & 45 \\
\hline 1 & 4 & 44 \\
\hline 2 & 1 & 11 \\
\hline \multicolumn{3}{|l|}{ HPV status } \\
\hline positive & 1 & 11 \\
\hline negative & 8 & 89 \\
\hline
\end{tabular}

immune responses [8-10]. We have previously shown that PDT has a significant impact on the expression of cancer testis antigens (CTA) in human HNSCCs and on the functionality of immune cell populations in blood samples of patients [11].

The process of epithelial-to-mesenchymal transition (EMT), also referred to as "mesenchymalization", involves a series of molecular and genetic changes in tumor cells that culminate in a conversion of the epithelial phenotype to a highly aggressive and invasive mesenchymal state [12, 13]. The change in cellular morphology that occurs during this conversion is characterized by a loss of cell adhesion and the acquisition of the migratory, invasive signature that favors cancer [14]. The molecular and genetic species that characterize EMT include transcription factors such as Snail, Slug, ZEB1, Twist and others $[15,16]$, changes in expression levels of cell surface proteins, such as a loss of E-cadherin and the appearance of $\mathrm{N}$-cadherin, expression of novel micro RNAs and changes in cytoskeletal proteins [13]. Effects of PDT on the EMT progression in various cancer types have been controversial [17-22]. For example, Della Pietra et al. reported that sub-optimal levels of PDT can induce EMT in prostate cancer cells [23], whereas Mao et al. showed that PDT combined with carboplatin suppressed EMT in laryngeal carcinomas [24]. The mechanisms responsible for these effects of PDF on the course of EMT remain unclear.

Tumor-derived exosomes (TEX) are cell derived nano-sized extracellular vesicles that play a crucial role in intercellular communication and in reprogramming of the tumor microenvironment (TME) [25]. We and others have reported that HNSCC are strong TEX producers [26] and that the plasma of patients with HNSCC is highly enriched in TEX [27]. TEX carry numerous immunosuppressive proteins as well as molecules promoting EMT [27-29]. Emerging evidence suggests that TEX can modulate EMT in carcinomas [30]. Franzen et al. [28] showed that urothelial cells treated with exosomes produced by bladder cancer increased expression levels of mesenchymal markers, e.g., alpha-smooth muscle actin ( $\alpha$ SMA), while down-regulating expression levels of epithelial markers such as E-cadherin as well as increasing their migration and invasion capabilities. Further, Min et al. reported that the exosome cargo of esophageal carcinoma cell lines was altered following radiation therapy, and that these altered exosomes promoted tumor metastasis by EMT induction [29]. Taken together, current reports indicate that: (a) cancer therapies can influence the morphology and behavior of tumor cells, in some cases promoting rather than inhibiting EMT and (b) tumor-derived exosomes are involved in the regulation of EMT, reflecting the parent cell responses to treatment regiments. However, most of the studies reporting exosome participation in EMT have been performed with cell lines or in mouse models. To the best of our knowledge, there is no study addressing the in vivo influence of oncological therapies on the ability of tumor-derived exosomes to modulate EMT in patients undergoing treatments.

This is the biggest cohort of HNSCC patients undergoing PDT with a standardized sample acquisition. Here, we show for the first time that exosomes isolated from plasma of patients with HNSCC treated with and responding to $\mathrm{PDT}$ recapitulate molecular characteristics 
of the parent tumors, serving as markers of PDT-induced molecular changes in the parent tumor. Moreover, these exosomes upon co-incubation with carcinoma cells can reverse the EMT phenotype of recipient cells and suppress their migration as well as invasiveness.

\section{RESULTS}

\section{Characteristics and molecular content of the plasma-derived exosomes prior to and after PDT}

Extracellular vesicles (EVs) isolated by size exclusion chromatography from plasma of HNSCC patients treated with PDT meet the criteria attributed to exosomes (Supplementary Figure 1). They have a size range of 30-150nm based on DLS and NTA analyses. Transmission electron microscopy (TEM) images show their vesicular morphology and confirm the vesicle diameter approaching $100 \mathrm{~nm}$. Western blots indicate the presence of Tsg101, an endocytic marker, and CD63, a tetraspanin, in the exosome cargo.

Exosomes were isolated from plasma specimens obtained prior to (t1) and serially at 3 different tine points ( $\mathrm{t} 2, \mathrm{t} 3$ and $\mathrm{t} 4)$ after PDT. The protein content was measured in all exosome fractions. The mean protein level in exosome fractions isolated from pre-therapy plasma specimens (t1) was $161 \mu \mathrm{g} / \mathrm{mL}$ (Figure 1). Following PDT, protein concentrations steadily decreased, reaching the mean level of $72 \mu \mathrm{g} / \mathrm{mL}$ at $\mathrm{t} 4$. The decreasing protein levels in serially collected exosome fractions from plasma of patients responding to PDT suggest that the total exosome protein might serve as an indication of response to this therapy in HNSCC patients.

The cargos of plasma exosomes obtained prior to and at the specified time points after PDT were evaluated by on-bead flow cytometry using total exosome fractions isolated from plasma. The exosome cargo was found to contain N-Cadherin (Figure 2A and 2B). The N-Cadherin levels were highest in the pre-therapy ( $\mathrm{t} 1)$ exosomes, and they decreased in exosomes isolated from the patients' plasma after PDT $(\mathrm{t} 3, \mathrm{t} 4)$. We also measured levels of E-Cadherin in the exosome cargo and found that following PDT, the E-Cadherin content increased to reach the highest level in exosomes collected at t4, i.e. 4-6 weeks after PDT (Figure 2A and 2B). The observed decreases in $\mathrm{N}$-Cadherin and corresponding increases in E-Cadherin levels in exosome fractions following PDT suggested that these changes might reflect PDT-induced reversal of EMT occurring in parent tumor cells.

Since it has been reported that TGF- $\beta 1$ is one of the main promoters of EMT [31], we also investigated levels of TGF- $\beta 1$ in the cargo of total exosomes obtained from pre- and post PDT plasma of all the patients. We found that exosomes isolated from pre-PDT plasma contained high levels of TGF- $\beta 1$, and that following PDT, these levels significantly decreased to reach the lowest levels in exosomes harvested at t4 (4-6 weeks after PDT) as shown in Figure 2C and 2D.

\section{Exosomes isolated from plasma of HNSCC patients undergoing PDT induce phenotypic changes in recipient cancer cells}

Previous studies have demonstrated that high expression levels of mesenchymal markers, such as Vimentin, Snail or Twist, are associated with poor prognosis in HNSCC [32]. To determine whether exosomes obtained from plasma of the HNSCC patients treated with PDT could induce expression of mesenchymal markers in epithelial tumor cells such as PCI13 and A549, co-incubation experiments were next performed.

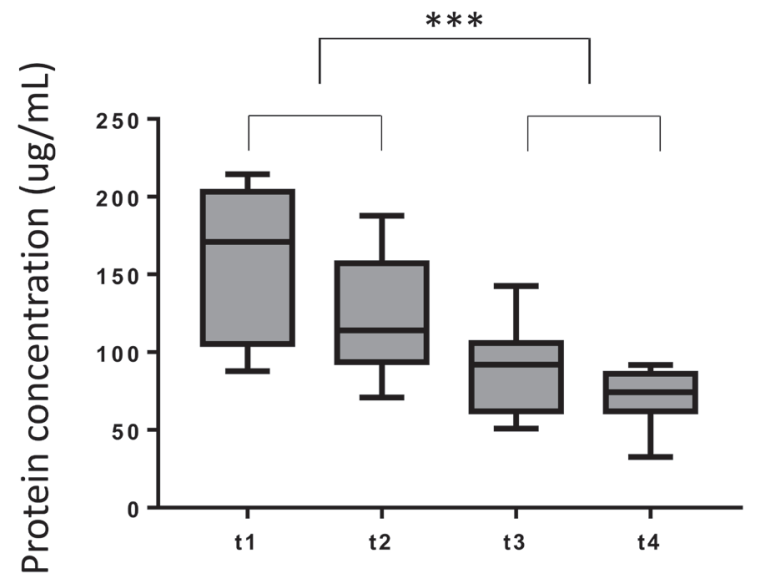

Figure 1: Protein concentrations of plasma-derived total exosome fractions for all HNSCC patients and for all time points $(\mathbf{n}=9)$. Exosomes harvested from patients before and immediately after PDT (t1 and t2) have a significantly higher protein concentration than exosomes harvested at the later time points after PDT ( $\mathrm{t} 3$ and $\mathrm{t} 4$ ). 2-tailed paired $\mathrm{t}$-test, $* * * \mathrm{p}<0.0001$. 
Total exosomes isolated from plasma at different time points pre- and post PDT were co-incubated with tumor cells as described in Methods. Total mRNA was isolated from tumor cells treated with exosomes and qRT-PCR was performed to evaluate gene expression of Vimentin, $N$-Cadherin, Snail, Twist, Slug and ZEB-1 in recipient cells. As shown in Figure 3, exosomes isolated from plasma prior to PDT induced the highest expression of these genes in the recipient tumor cells. In contrast, exosomes obtained after PDT, progressively lost the ability to induce transcription of these genes in the recipient cells.
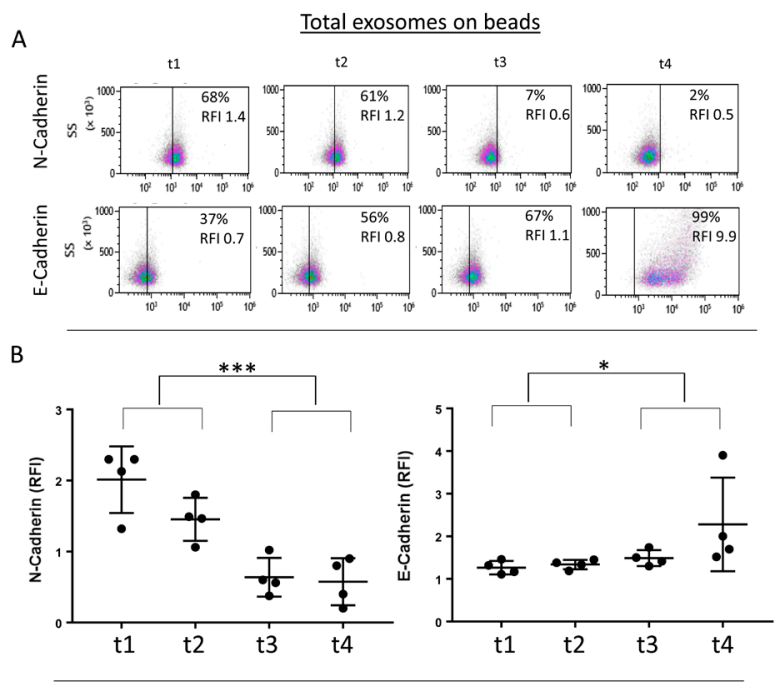

C

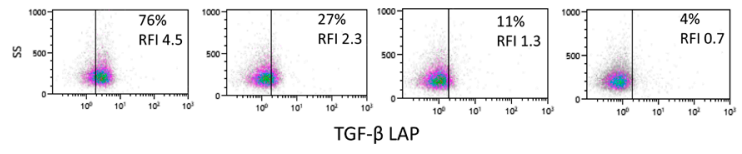

D

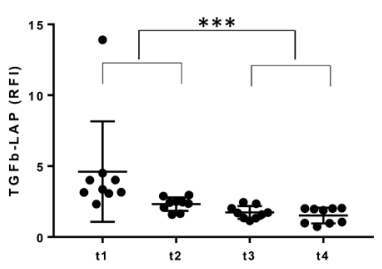

Figure 2: Alterations in the molecular cargo of exosomes harvested from HNSCC patients undergoing PDT. (A) Representative flow cytometry density plots for E-Cadherin and N-Cadherin levels carried on total exosomes harvested at $\mathrm{t} 1, \mathrm{t} 2, \mathrm{t} 3$ and $\mathrm{t} 4$. Results are presented as RFIs as well as \% positive exosomes. (B) Combined flow cytometry results are shown for 4 patients. Note the decreasing levels of N-Cadherin during therapy compared to the increasing levels of E-Cadherin. (C) Representative and combined (D) data for levels of TGF- $\beta 1$ carried on exosomes harvested from HNSCC patients undergoing PDT $(n=9)$. 2-tailed paired t-test, $* \mathrm{p}=0.007 ; * * * \mathrm{p}<0.0002$.
Further, pre-PDT exosomes failed to induce the mRNA transcript for E-Cadherin in the same recipient tumor cells. However, exosomes harvested after PDT (on day 7 or week 4-6) significantly up-regulated mRNA transcript for $E$-Cadherin. The latter effect was especially prominent in A549 cells. There was no significant upregulation of ZEB-1 mRNA in PCI-13 cells co-incubated with exosomes as opposed to significant up-regulation of ZEB-1 message in A549 cells indicating variability in responses to exosome uptake by different tumor cells (Figure 3 ).

To determine whether transcriptional changes induced by exosomes from plasma of HNSCC patients translate to protein expression in PCI-13 and A549 cells, $72 \mathrm{~h}$ co-incubation with exosomes was followed by immunofluorescence and confocal microscopy (Figure 4A and B). Pre-PDT exosomes completely down-regulated expression of EpCAM and concomitantly up-regulated Vimentin expression in PCI-13 and A549 cells. Exosomes harvested from plasma at $\mathrm{t} 2$ (24h after PDT) induced similar effects. In contrast, exosomes obtained at $\mathrm{t} 3$ and t4 after PDT induced strong expression of EpCAM and down-regulated Vimentin in both recipient cells (Figure 4).

These experiments show that plasma-derived exosomes of the HNSCC patients undergoing PDT when co-incubated with tumor cells induced transcriptional and translational alterations in the key components of the EMT pathway in the recipient cells. Further, while exosomes isolated prior to or immediately after PDT increased expression of mesenchymal proteins, those harvested from plasma after PDT, at the time corresponding to PDT-induced clinical responses, increased expression of epithelial markers and lost mesenchymal Vimentin.

\section{Exosomes isolated from plasma of HNSCC patients undergoing PDT induce functional changes in recipient tumor cells}

EMT has been shown to be associated with cancer invasion and metastasis in several malignancies, including HNSCC [33-35]. We, therefore, expected that plasma exosomes of HNSCC patients undergoing PDT that induced EMT-related phenotypic changes in recipient cells might also alter their functions. Indeed, exosomes harvested from plasma at $\mathrm{t} 1$ and $\mathrm{t} 2$ strongly promoted proliferation of recipient tumor cells (Figure 5A), while those harvested at $\mathrm{t} 3$ and $\mathrm{t} 4$ lost the ability to promote tumor growth. Similarly, spheroid formation by tumor cells was augmented by the pre-PDT and $24 \mathrm{~h}$ post-PDT exosomes but was no longer supported by exosomes collected at $\mathrm{t} 3$ and $\mathrm{t} 4$ (Figure 5B). The data suggest that tumor cell growth is only promoted by exosomes produced when the tumor has the mesenchymal phenotype, and it ceases to be promoted with cell conversion to the epithelial phenotype. Not only tumor cell proliferation but also motility and chemotaxis of tumor cells are differentially 
modulated by exosomes from plasma of HNSCC patients undergoing PDT (Figure 6A and 6B and supplementary Fig.2).

To further determine the functional changes in tumor cells induced by plasma-derived exosomes, invasive abilities of cancer cells co-incubated with exosomes harvested at different time points during PDT were investigated in Matrigel-based migration assays (Figure 6C and D). While control cells were minimally invasive, E-Cadherin loss and Vimentin upregulation resulted in a significant increase in both motility and invasiveness in cancer cells pretreated with $\mathrm{t} 1$ and $\mathrm{t} 2$ exosomes. In contrast, co-incubation with $\mathrm{t} 3$ and $\mathrm{t} 4$ exosomes did not enhance tumor cell invasiveness, remaining at the level of invasion mediated by exosomes from plasma of normal controls.

\section{DISCUSSION}

Epithelial cells are characterized by the ability to establish close connections with neighboring cells. Malignant transformation of epithelial cells induces a change of their phenotype culminating in a loss of cell adhesion and the acquisition of an invasive and migratory phenotype [13]. As the cargo of exosomes derived from cultured tumor cells or from plasma of cancer patients has $\mathrm{PCl} 13$

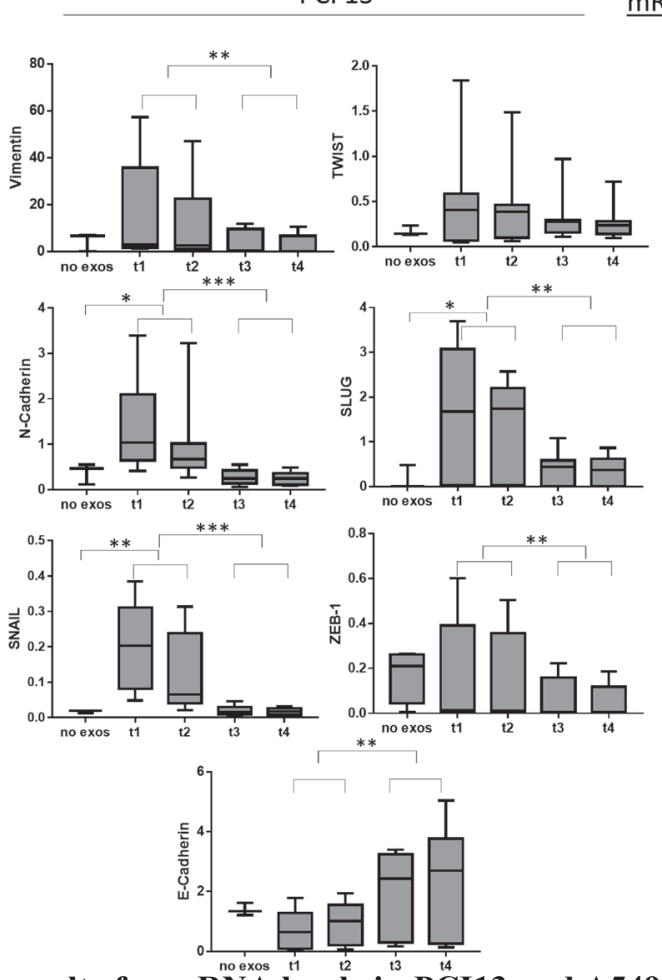

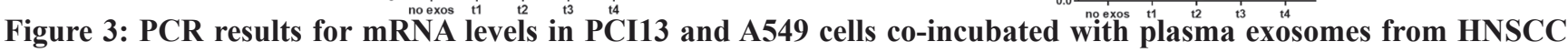
patients undergoing PDT. Exosomes harvested at all four time points were harvested and co-incubated with tumor cells (n=9). Following co-incubation, qRT-PCR using mRNA obtained from recipient cells was performed as described in Methods. Results are presented as fold increases using the housekeeping gene, HPRT1 as a reference gene. Note the increased expression levels of mesenchymal mRNA for Snail, Slug, Twist, Vimentin, N-Cadherin and Zeb1 at t1 and t2. The epithelial marker, E-Cadherin, was significantly increased at $\mathrm{t} 3$ and $\mathrm{t} 4$. Cancer cells co-incubated with no exosomes were used as negative controls (-). 2-tailed paired $\mathrm{t}$-test, ${ }^{*} \mathrm{p}<0.05$; ${ }^{* *} \mathrm{p}<0.004$; $* * * \mathrm{p}<0.0001$.

been shown to contain various EMT-relevant molecules [30], it has been suggested that tumor-derived exosomes (TEX) present in plasma of patients with cancer might play a role in the regulation EMT progression. In this study, we tested this hypothesis by contrasting the effects induced by TEX isolated from serially collected plasma specimens of HNSCC patients responding to PDT.

Various biological pathways are known to promote EMT, regulating expression levels of cytoskeletal molecules or activating the EMT-relevant transcription factors. Cadherins are adhesion proteins that regulate the balance between suppression and promotion of tumor cell invasion. E-Cadherin functions as a suppressor of invasion and is downregulated in various cancers including HNSCC in general $[36,37]$ and in the patients in this study evaluated prior to or immediately after receiving PDT. While the cohort of patients we evaluated was small, all the patients in our study cohort had highly aggressive, therapy-refractive tumors, where a mesenchymal profile is expected. In contrast to E-Cadherin, N-Cadherin promotes migration and invasion, is often upregulated in advanced cancers and its expression correlates with poor prognosis in HNSCC $[37,38]$. Transcriptional factors such as Slug, Snail and Twist are known to be the downstream targets of signaling pathways operating in EMT in concert with suppression of the epithelial profile, including that of

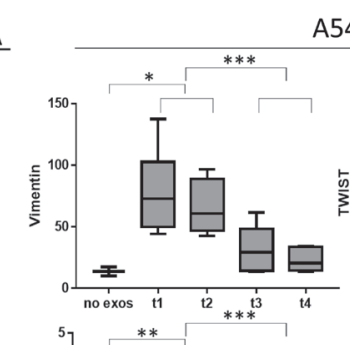
549
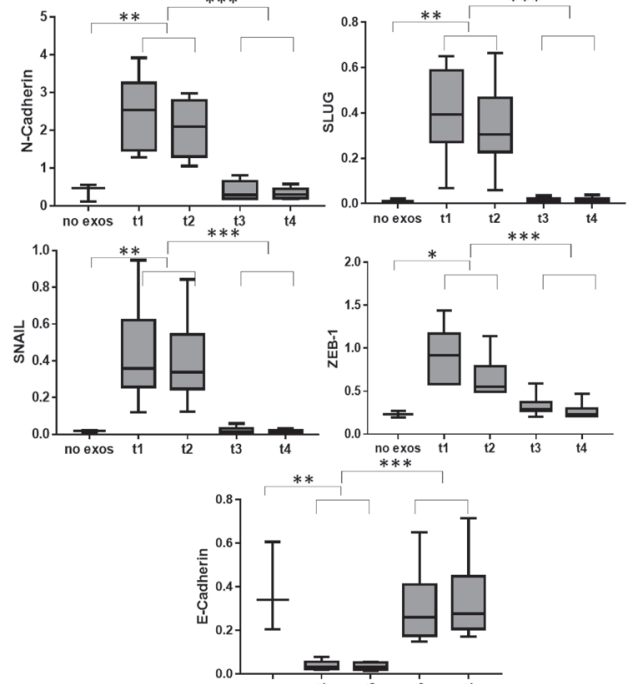
E-Cadherin [39, 40]. Moreover, an inverse relationship between Snail and E-cadherin has been noted to associate with patients' outcome in breast cancer and oral squamous cell carcinoma [41, 42]. For these reasons, levels of expression of mRNAs coding for these molecules in recipient tumor cells co-incubated with TEX was of special interest.

Using exosomes isolated from plasma of the HNSCC patients undergoing PDT, all of whom experienced a clinical benefit after therapy, we demonstrated that these exosomes: (a) carried a cargo of molecules and genes that recapitulated the EMT progression/regression of the parental tumor occurring in response to PDT; and (b) effectively induced EMT progression/regression in recipient tumor cells following ex vivo co-incubation. The inverse effects mediated by $\mathrm{t} 1 / \mathrm{t} 2$ vs $\mathrm{t} 3 / \mathrm{t} 4$ plasmaderived exosomes obtained from the same patients' plasma convincingly illustrate the dual capability of these exosomes to either promote or reverse mesenchymalization in recipient tumor cells. This dual potential of exosomes to modulate EMT is clearly dependent on the cargo they carry, which presumably reflects the changing molecular/ genetic content of the parent tumor cells responding to PDT. Thus, exosomes, which in cancer patients are likely to be enriched in TEX, emerge as potential biomarkers of the tumor response to therapy. Further, these plasmaderived exosomes are biologically active and carry a cargo of molecules/genes that induce mRNA and protein alterations in recipient tumor cells. The phenotypic and functional alterations driven by plasma-derived exosomes can either promote or suppress EMT in recipient cells. In patients with active disease refractory to therapy, plasmaderived exosomes serve to promote EMT. However, in the HNSCC patients responding to PDT, plasma-derived exosomes suppressed EMT progression in recipient tumor cells. In this paradigm, exosomes, and especially TEX that phenotypically and functionally recapitulate parent tumor cells, appear to act as non-invasive predictors of response to PDT on the one hand and as conveyors of messages that can modulate the EMT program on recipient cells on the other.

We and others have previously shown that PDT influences patients' outcome by promoting anti-tumor immune responses [43, 44]. Regression of distant metastasis after PDT of primary lesions has also been reported [45]. In this study, we provide evidence that PDT can also modulate the TME in human cancers through the exosome-mediated reversion of the mesenchymal profile and reducing the aggressiveness and invasiveness of the cancer. The involvement of exosomes isolated from plasma of HNSCC patients treated with PDT in this process introduces a new and highly effective biological communication strategy that promises to be useful in future monitoring of responses of cancer patients to oncological therapies.

While this retrospective study has limitations due to a small patient numbers and the paucity of experiments investigating the mechanistic insights of TEX-mediated effects, its merit lies in the demonstration that serial monitoring of the exosome cargo in patients' plasma during the delivery of anti-cancer therapy informs about the therapy-induced changes in the tumor and hence about outcome.

\section{MATERIALS AND METHODS}

\section{Study design}

Nine HNSCC patients were treated with photodynamic therapy using Temoporfin (Foscan $^{\circledR}$, Biolitec, Austria) in a palliative setting due to recurrence of cancer between 2014 and 2016, as previously described [11]. Peripheral blood samples of all patients were collected after control of the patient's hemoglobin level at four different time points: 24 hours before PDT (t1), 24 hours after PDT (t2), one week after PDT (t3) and 4 to 6 weeks after PDT (t4). All patients had undergone various therapeutic treatments such as primary radio-chemotherapy and/or surgery according to the oncologic guidelines. PDT treatment was performed in general anesthesia, and the patients were hospitalized for a minimum of $7 \mathrm{~d}$ with decreasing light restrictions. Table I provides information on the clinicopathological characteristics of these patients and additional information can be also found in reference. [11]. The follow-up period was from 5 to 112 months, with a mean of 43.6 months. Seven patients were cancer free and 2 patients still had a tumor, but the tumor load was decreased. The overall survival of all patients was 45 months.

Peripheral blood was also obtained from five healthy donors. Plasma samples were used for exosome isolation to serve as controls in experiments using patient-derived plasma exosomes. At least three biological replicates were performed for each experiment with the exact numbers specified in figure legends.

\section{Statistics}

Analysis were conducted using GraphPad Prism 5 software. Comparison between continuous variables was performed using the 2 -tailed paired t-test. $\mathrm{P}<0.05$ was considered significant. Overall comparison between different time points was performed using one-way Anova.

\section{Collection of plasma}

After isolation of PBMCs as described [11], plasma was centrifuged for $10 \mathrm{~min}$ at $2,500 \mathrm{xg}$ and collected for storage at $-20^{\circ} \mathrm{C}$. Plasma samples were thawed 

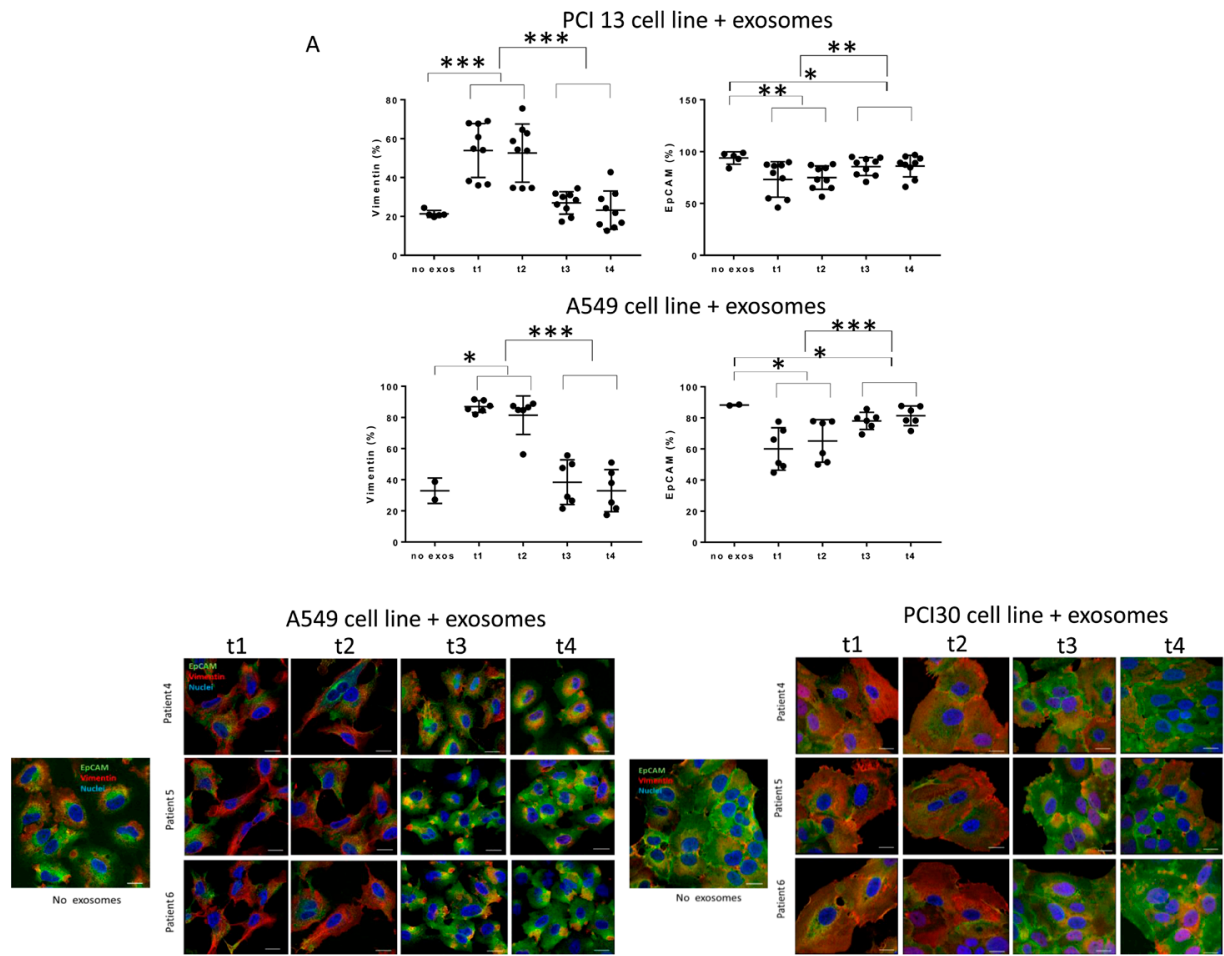

Figure 4: Alterations in expression levels of EpCAM or Vimentin in PCI-13 and A549 cells co-incubated with plasma exosomes from HNSCC patients undergoing PDT. Exosomes harvested at all four time points were co-incubated with tumor cells. Staining for EpCAM or Vimentin expression on the recipient cells was performed and cells were examined by flow cytometry in (A) or by confocal microscopy in (B). Note the decreasing levels of Vimentin at $\mathrm{t} 3$ and $\mathrm{t} 4$ and the simultaneously increasing EpCAM levels. Note also that the morphology of A549 cells appears altered to cuboidal after co-incubation with exosomes obtained at $\mathrm{t} 3$ and $\mathrm{t} 4$, while treatment with exosomes from $\mathrm{t} 1$ and $\mathrm{t} 2$ induces a spindle-like morphology (in B). Scale bar $=20 \mu \mathrm{m}$. 2-tailed paired t-test, ${ }^{*} \mathrm{p}<0.05 ; *^{*}<0.004$; $* * * p<0.0001$. Cancer cells incubated with medium alone were used as negative controls (no exos).

A

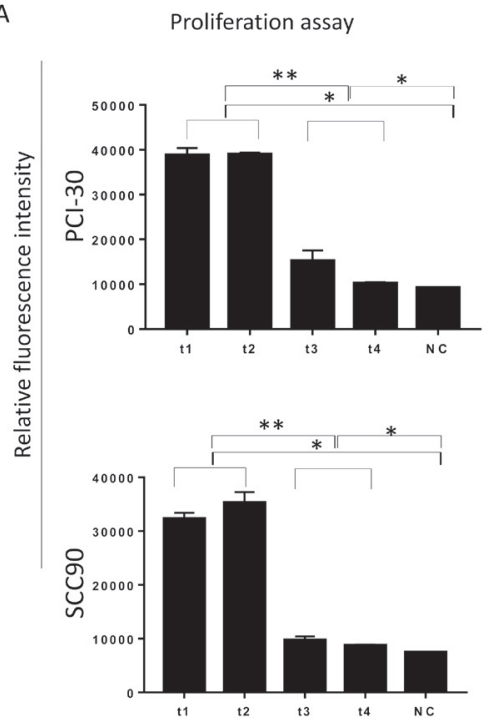

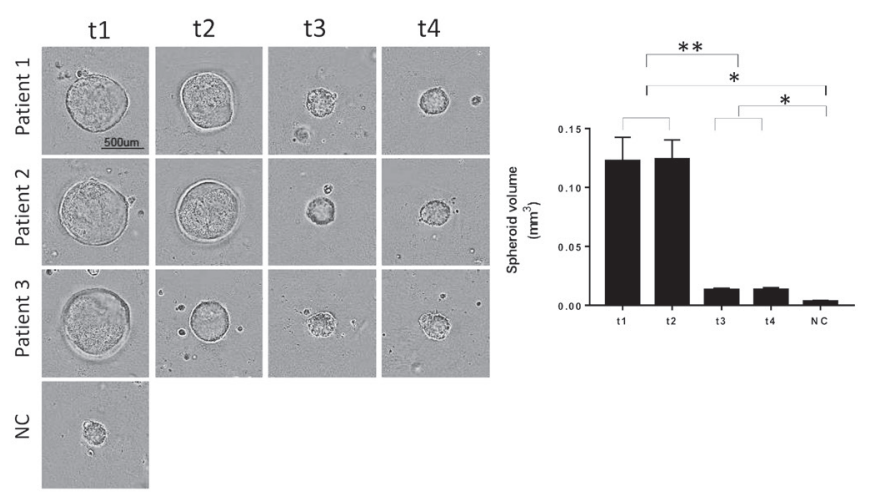

Figure 5: Proliferation of cancer cells co-incubated with plasma exosomes obtained from HNSCC patients undergoing PDT. (A) Proliferation assay using PCI-30 and SCC90 cells incubated \pm exosomes from plasma of HNSCC patients ( $=3$ ). Exosomes from all 4 time points were tested. In A, results are presented as relative fluorescence intensity. Proliferation of cancer cells was increased after co-incubation with $\mathrm{t} 1$ and $\mathrm{t} 2$ exosomes, whereas cells co-incubated with $\mathrm{t} 3$ and $\mathrm{t} 4$ exosomes behave like the untreated controls. In (B), the 3D spheroid assay with PCI-30 cancer cells co-incubated with total exosomes from different time points before and after PDT (n=3). Note the significantly higher spheroid volume after co-incubation with $\mathrm{t} 1$ and $\mathrm{t} 2$ exosomes. In contrast, $\mathrm{t} 3$ and $\mathrm{t} 4$ exosomes behaved like negative controls. 2-tailed paired t-test, $* \mathrm{p}<0.02 ; * * \mathrm{p}<0.002$. 

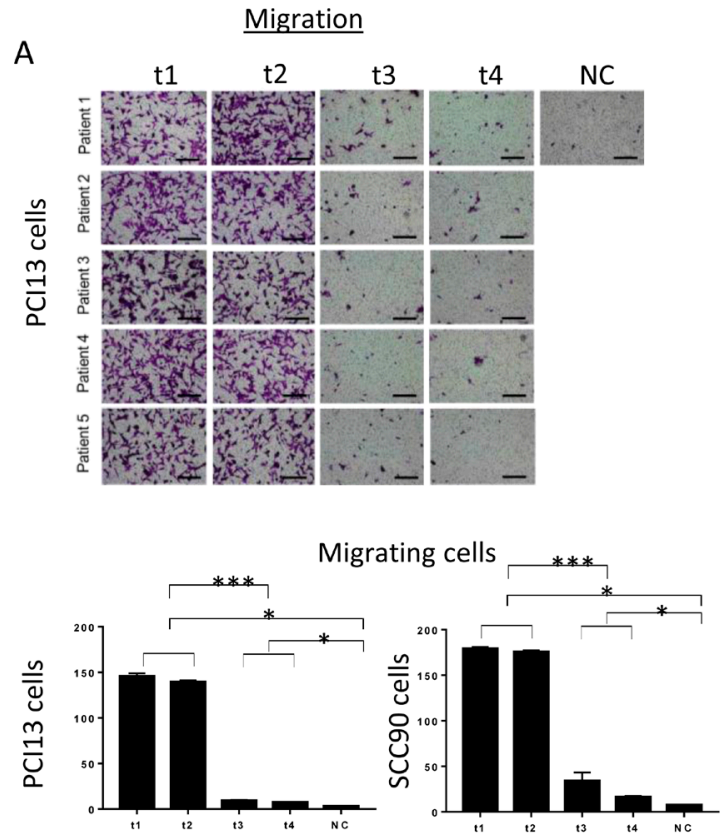

C

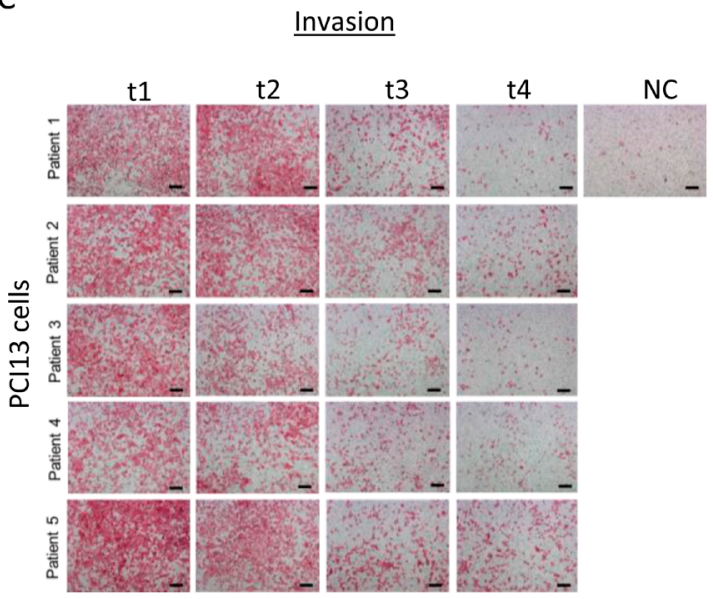

B
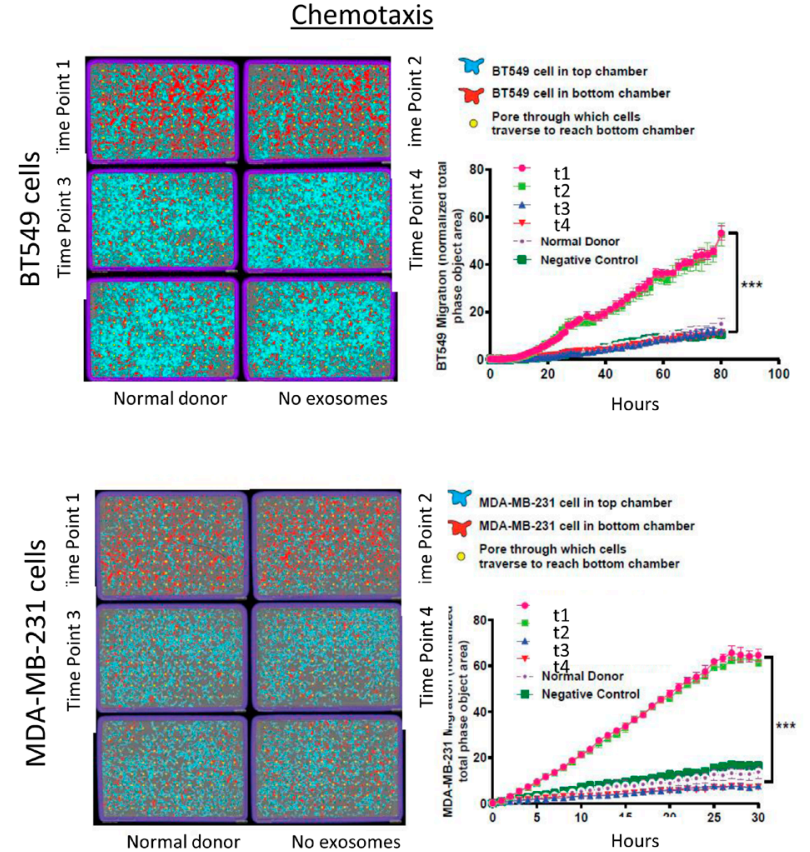

D

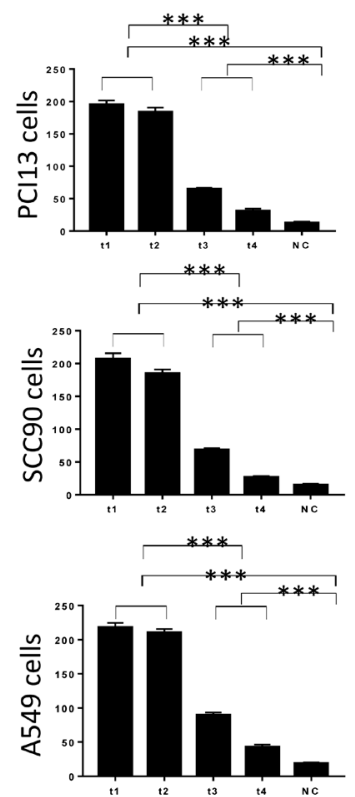

Figure 6: Migration, chemotaxis and invasiveness of tumor cells co-incubated with plasma exosomes obtained from HNSCC patients undergoing PDT. In (A), representative and combined results of migration of PCI13 cells treated with total exosomes of 5 HNSCC patients. Note that $\mathrm{t} 1$ and $\mathrm{t} 2$ exosomes increased migration in tumor cells, whereas no migration was observed with tumor cells co-incubated with $\mathrm{t} 3$ and $\mathrm{t} 4$ exosomes. Scale $\mathrm{bar}=50 \mu \mathrm{m}$. In (B), representative results of the chemotaxis assay using two different cell lines, BT549 and MDA-MB-231 cells. Total exosomes of patient \#2 obtained before and after PDT were used as chemoattractant. The data for exosomes of two additional patients are in Supplementary Figure 2. Chemotaxis towards $t 1$ and $t 2$ exosomes is significantly greater than that toward t3 and t4 exosomes. The graphs demonstrate exosome-driven chemotaxis (patients' vs normal controls' exosomes and no exosome controls) of cancer cells over time. In (C), representative results of the invasion assay using PCI13 cell line. Invasion of cancer cells co-incubated with $\mathrm{t} 1$ and $\mathrm{t} 2$ was significantly greater than that with $\mathrm{t} 3$ and $\mathrm{t} 4$ exosomes. Scale bar $=200 \mu \mathrm{m}$. In (D), combined results for all three cell lines, PCI13, SCC90 and A549 used in invasion assays performed with pre- and post-PDT exosomes of 5 HNSCC patients. 2 -tailed paired t-test and one-way Anova, ${ }^{*} \mathrm{p}<0.02,{ }^{* * *} \mathrm{p}<0.0007$. 
and batched, so that all samples of every patient were assayed together to avoid inter-measurement errors. Clinicopathological information for all patients can be found in our previous publication [11].

\section{Exosome isolation by mini size-exclusion chromatography (mini-SEC)}

The mini-SEC method for exosome isolation was developed and optimized as previously described [46]. Briefly, plasma samples were thawed and centrifuged first at $2,000 \mathrm{xg}$ for $10 \mathrm{~min}$ at room temperature (RT) and then for $30 \mathrm{~min}$ at $14,000 \mathrm{xg}$ at $4^{\circ} \mathrm{C}$. Next, plasma was ultrafiltered using a $0.22 \mu \mathrm{m}$ filter (EMD Millipore, Billerica, MA, USA). An aliquot of plasma (1mL) was placed on a mini-SEC column and eluted with PBS. The void volume fractions \#3 - \#5 enriched in exosomes were collected ( $1 \mathrm{~mL} /$ fraction). The fraction \#4 contained the majority of isolated exosomes as previously reported [46] and was used for all exosome studies. The fraction \#4 exosomes are referred to as "total exosomes."

\section{BCA protein assay and sample concentration}

The protein concentration in the fraction \#4 was measured using the Pierce BCA protein assay kit (Pierce Biotechnology, Rockford, IL, USA) according to the manufacturer's instructions. The protein concentrations were calculated as $\mu \mathrm{g}$ protein $/ 1 \mathrm{~mL}$ plasma. Concentration of $1 \mathrm{~mL}$ specimens was performed using Vivaspin 500 (VS0152, 300,000 MWCO, Sartorius, Göttingen, Germany).

\section{Exosome Characterization}

\section{Dynamic Light Scattering}

Size measurements of exosomes were done using a Zetasizer (Malvern Instruments Ltd, England, UK). Exosomes diluted in PBS (1:100) were analyzed in equilibration time of $120 \mathrm{sec}$ at the constant temperature of $25^{\circ} \mathrm{C}$.

\section{Nanotracking analysis}

Exosomes were diluted to an appropriate level with particle-free PBS and continuously fed into the Nanoparticle Tracking Analysis (NTA) (NanoSight, Amesbury, UK) LM-10 system with a syringe pump. The Brownian motion of each individual exosomes within the field of view was visualized with a laser illumination unit and a high-definition CCD camera. Each measurement was recorded for $1 \mathrm{~min}$ and repeated for three times. The size distribution of exosomes was then analyzed and extracted from the motion of exosomes using the software that came with the NTA system.

\section{Transmission Electron Microscopy}

Isolated total exosomes were fixed with 4\% glutaraldehyde (Electron Microscopy Services, Hatfield, PA, USA) for $20 \mathrm{~min}$ at RT. A $10 \mu \mathrm{L}$ droplet of glutaraldehyde-fixed exosomes was placed on Formvarcoated 300 mesh copper grid (Electron Microscopy Services, Hatfield, PA). The sample was incubated for $1 \mathrm{~min}$ followed by rinsing with DI water for $1 \mathrm{~min}$ to ensure removal of PBS salts. Excess liquid was blottedoff with a Whatman filter. Post rinsing, $50 \mu \mathrm{l}$ of Uranylacetate solution was put on the grid and allowed to remain for $1 \mathrm{~min}$. Excess liquid was removed, and the grids were viewed on a Hitachi H-7100 transmission electron microscope (TEM, Hitachi High Technologies) operating at $100 \mathrm{keV}$. Digital images were collected using an AMT Advantage 10 CCD Camera System (Advanced Microscopy Techniques) and inspected using NIH ImageJ software.

\section{Western blots}

Western blots for exosome proteins TSG101 and CD63 were performed as previously described [26].

\section{Detection of surface proteins on exosomes}

For detecting exosome-associated surface proteins, on-bead flow cytometry was performed following exosome capture on streptavidin magnetic beads coated with biotinlabeled anti-CD63 mAbs. The detailed protocol was previously reported [47]. The following labeled detection Abs were used for staining: anti-E-cadherin (Biolegend, \# 324104), anti-N-cadherin (Biolegend \# 350808) or and anti-TGF- $\beta$ (R\&D, \# FAB2463P). Immediately following 1h staining with Abs at RT and washing with buffer, flow cytometry was performed using a Gallios instrument. Samples were run for $2 \mathrm{~min}$ and 10,000 events were acquired. For each detection $\mathrm{Ab}$, corresponding isotype controls were included.

\section{Cell lines}

For all experiments, the following squamous cell carcinoma lines were used: PCI13, a HNSCC HPV-negative cell line and SCC-90, a HNSCC HPV+ cell line were established and maintained in our laboratory [48]. The other cell lines used: A549 (human lung adenocarcinoma), MDAMB231 (human breast adenocarcinoma) and BT549 (human breast ductal carcinoma) were obtained from the ATCC and were provided to us by Dr. Peter Lucas (Children's hospital, Pittsburgh). All cell lines were routinely tested for Mycoplasma and were contamination free. Cells were cultured in $150 \mathrm{~cm} 2$ cell culture flasks in $25 \mathrm{ml}$ of DMEM supplemented with $\alpha 1 \%(\mathrm{v} / \mathrm{v})$ penicillin and streptomycin and $10 \%(\mathrm{v} / \mathrm{v})$ exosome-depleted fetal bovine serum (FBS, 
Gibco, Fisher Scientific, Pittsburgh, PA) at $37^{\circ} \mathrm{C}$ in an atmosphere of 5\% CO2 in air. The cells were harvested upon reaching confluency of $60 \%$ to $80 \%$.

\section{TaqMan analysis of mRNA expression levels in exosomes}

To evaluate expression levels of EMT-related genes, TaqMan analysis was performed. Aliquots $\left(20 \times 10^{4}\right)$ of tumor cells were co-incubated with exosomes $(10 \mu \mathrm{g}$ protein) for $96 \mathrm{~h}$ and cells were then harvested using RLT buffer (Qiagen, Hilden, Germany). RNA was extracted using an RNeasy Kit (Qiagen). The RNA was used for cDNA synthesis and expression analysis by TaqMan in the StepOnePlus system (Applied Biosystems, CA, USA) was performed. Commerciallyavailable TaqMan primers (Thermo Fisher Scientific Life Technologies, MA, USA) were purchased to evaluate local expression of the key mesenchymal and epithelial markers Vimentin, E-Cadherin, N-Cadherin, Slug, Snail, Twist, ZEB-1 and $\alpha$-SMA (see Supplementary Table 1 for a list of all assay IDs). The expression of each gene was normalized to the HPRT1 housekeeping gene and calculated as fold increase [49].

\section{Flow Cytometry of tumor cells}

\section{Detection of surface and intracellular proteins in cancer cells}

Aliquots of cell lines, PCI13 and A549 (50x10 ${ }^{4}$ cells/stain suspended in FACS buffer) were co-incubated with exosomes for $96 \mathrm{~h}$ and were harvested by using cold PBS. For surface staining, non-specific binding was blocked by using a house-made blocking buffer ( $1 \%$ BSA, $1 \%$ human serum albumin (HSA) in PBS) for $15 \mathrm{~min}$. Incubation with the appropriate labeled Abs specific for Vimentin; Abcam \#ab128507 and EpCAM; Abcam \#ab187293) was performed for $1 \mathrm{~h}$. For intracellular staining, cells were first treated with FIX-Perm buffer (Biolegend, \#421403) for 40min at 40C. After washing with Perm buffer and blocking of non-specific binding, staining with Vimentin conjugated $\mathrm{Ab}$ was performed for $30 \mathrm{~min}$ at RT. Flow cytometry was performed using a Gallios instrument equipped with Kaluza 1.0 software (Beckman Coulter, Krefeld, Germany). Isotype controls were included in all flow cytometry experiments.

\section{Immunofluorescence}

Tumor cells $\left(0.1 \times 10^{6}\right)$ were plated on collagen type I-coated $18 \mathrm{~mm}$ coverslips and allowed to adhere overnight. Next day, fresh medium containing $20 \mu \mathrm{g} / \mathrm{mL}$ of exosomes was added. After $72 \mathrm{~h}$ co-incubation, cells were washed in PBS, fixed in $3.33 \%$ PFA for $20 \mathrm{~min}$ at RT and were permeabilized with $0.1 \%$ TritonX (MilliporeSigma, St. Louis, MO, USA) for $10 \mathrm{~min}$. To minimize non-specific binding, blocking was performed with $10 \%$ goat serum for $20 \mathrm{~min}$ at RT. Cells were then rinsed with wash buffer (PBS, 0.1\%BSA) and incubated with 1:100 dilution (PBS with $0.1 \%$ BSA) of primary antibodies: mouse anti-human Vimentin (Ab8069; Abcam, Cambridge, MA) and rabbit anti-EpCAM (Ab71916; Abcam, Cambridge, MA) overnight at 4oC. Cells were rinsed $3 \mathrm{x}$ with wash buffer and incubated with 1:500 (PBS, 1\% BSA) dilution of secondary antibodies: goat anti-mouse Dylight $488 \mathrm{~nm}$ (4408S, Cell Signaling Technologies, Danvers, MA, USA) and goat anti-rabbit Dylight 647 nm (4414S, Cell Signaling Technologies, Danvers, MA) for $2 \mathrm{~h}$ at RT. Lastly, cells were rinsed $5 \mathrm{x}$ with wash buffer and imaged using a Zeiss LSM 880 confocal microscope (Carl Zeiss, Thornwood, NY, USA).

\section{Functional assays}

\section{Proliferation}

Proliferation was quantified by using direct CyQUANT nucleic acid-sensitive fluorescence assay (Thermo Fisher Scientific, Waltham, MA, USA) according to the manufacturer's instructions. Briefly, $200 \mu \mathrm{L}$ aliquots of cell suspension containing $1.25 \times$ $10^{3}$ cells $/ \mathrm{mL}$ were plated in wells of a 96-well microplate (Corning Inc., Corning, NY, USA) and allowed to adhere for $4 \mathrm{~h}$. Exosomes $(10 \mu \mathrm{g} / \mathrm{mL})$ were added to respective treatment wells and co-incubated with tumor cells for $72 \mathrm{~h}$. Next, cells were labeled with CyQUANT ${ }^{\circledR}$ Direct and fluorescence intensities were measured with TECAN spectrophotometer reader (\#TECAN, Männedorf, Switzerland). Proliferation was assessed by plotting relative fluorescence intensities.

\section{Spheroid assays}

3D-spheroid assays were performed following the IncuCyte 96-well Kinetic 3D Spheroid Protocol (Essen BioScience, Ann Arbor, MI, USA). Cells $\left(2.5 \times 10^{2} / 200 \mu \mathrm{L}\right.$ medium) were seeded in wells of Ultra Low attachment plates (Cat \# ULA7007, Corning Inc, Corning, NY, USA) and cultured in DMEM media containing 5\%FBS and 2\% Cultrex BME (Trivigen, Gaithersburg, MD, USA) to allow spheroid formation. After 3 days, $100 \mu \mathrm{L}$ of medium containing exosomes $(10 \mu \mathrm{g} / \mathrm{mL})$ was added to each well and spheroid growth was monitored for 12 additional days, replacing the medium every 3 days. Spheroid growth was monitored by phase contrast images taken every $24 \mathrm{~h}$ with a $10 \mathrm{X}$ objective. Spheroid volumes were calculated post 12 days using SpheroidSizer (MATLAB-based opensourced software). 


\section{Migration assay}

Migration assays were performed in in wells of 24 Transwell plates with an 8 - $\mu \mathrm{m}$ pore-size polycarbonate filter (\#ECM508; Millipore-Sigma, St. Louis, MO, USA). All media and supernatants were brought to room temperature before usage. The lower chamber contained $650 \mu \mathrm{L}$ of DMEM complete media $(10 \%$ FBS) supplemented with rhEGF (10ng/ml, AF-10015, Peprotech, Rocky Hill, NJ, USA) and the upper chamber contained PCI13 or A549 cells ( 1 X $10^{5}$ cells in $10 \mu \mathrm{L}$ of medium with $2 \%$ FBS in media) pretreated with or not pretreated with exosomes for 96h. After an incubation period of $36 \mathrm{~h}$, the cells in the upper chamber (non-migrated cells) of the Transwell were removed, and the cells migrated to the lower side of the membrane were fixed with $4 \%$ glutaraldehyde for $10 \mathrm{~min}$ and stained with $0.2 \%$ crystal violet for $10 \mathrm{~min}$ at RT. Five random bright-field images were taken per Transwell membrane using a $5 \mathrm{X}$ objective of a Zeiss Axiovert 200M microscope (Carl Zeiss Microimaging, Thornwood, NY, USA). The number of migrated cells per condition was manually counted and subtracted from the number of cells migrated towards plain medium alone.

\section{Chemotaxis assays}

Chemotaxis assays were performed using IncuCyte ClearView 96-well \#137 plates (Essen BioScience) containing Transwell membranes (\#143) with $8 \mu \mathrm{M}$ diameter pores. Next, $2.5 \times 10^{3}$ tumor cells (MDAMB231 or BT549 cells) suspended in $6 \mu \mathrm{L}$ of $0.5 \%$ FBS were placed in the upper chamber and allowed to adhere to the membrane surface for $30 \mathrm{~min}$ at RT. Exosomes ( $2 \mu \mathrm{g}$ protein) isolated from plasma of the HNSCC patients or normal donors were suspended in $200 \mu \mathrm{L}$ of medium containing $1 \%(\mathrm{v} / \mathrm{v}) \mathrm{FBS}$ and placed in lower chambers of the Transwell plate. Images of cells collecting at the upper and bottom surfaces of each ClearView membrane were obtained every $2 \mathrm{~h}$ for $3 \mathrm{~d}$ using an IncuCyte live-cell Imaging System. The IncuCyte Chemotaxis Analysis software module was used to quantify migration of tumor cells towards medium containing plasma-derived exosomes.

\section{Invasion assays}

Migration assays were performed in in wells of 24 Transwell plates with 8-um pore-size polycarbonate filter (\#ECM508; Millipore-Sigma, St. Louis, MO). The 8-um pore-size polycarbonate filter was coated with $1 \mathrm{mg} / \mathrm{ml}$ Cultrex ${ }^{\circledR}$ RGF BME Type 2 (Trevigen, Gaithersburg, MD, USA). The lower chamber contained $650 \mu 1$ of DMEM media supplemented with $0.1 \%$ FBS and $10 \mathrm{ng} / \mathrm{ml} \mathrm{rhEGF}$ and the upper chamber had
1 X 105 cells $(100 \mu \mathrm{L}$ of $0.1 \%$ FBS media) of either PCI13, A549 or SCC90 cells pretreated with or without exosomes for $96 \mathrm{~h}$. After an incubation period of $36 \mathrm{~h}$, non-migrating cells in the top chamber Transwell membranes were removed and membranes were fixed and stained according to the Diff-Stain Kit staining protocol (Cat No: K7128, IMEB Inc., San Marcos, CA, USA). The quantification and analysis were performed as described above (Migration assays).

\section{Study approval}

The acquisition of blood samples (patients and healthy controls) and clinical data for research purposes, was approved by the local ethics committee (\# 323/14, University of Ulm, Germany). A written informed consent of all patients and healthy controls was received prior to inclusion in this study.

\section{Abbreviations}

$\begin{array}{ll}\text { aSM } & \text { Aalpha-smooth muscle actin } \\ \text { CTA } & \text { cancer testis antigens } \\ \text { EMT } & \text { epithelial-to-mesenchymal transition } \\ \text { EVs } & \text { extracellular vesicles } \\ \text { HNSCC } & \text { head and neck squamous cell carcinoma } \\ \text { PDT } & \text { photodynamic therapy } \\ \text { TEM } & \text { ransmission electron microscopy } \\ \text { TEX } & \text { tumor-derived exosomes } \\ \text { TME } & \text { tumor microenvironment }\end{array}$

\section{Author Contributions}

M-NT and SY performed experiments and wrote the manuscript. TKH and JT reviewed the manuscript; CB provided the samples and organized shipment; TLW designed the study, interpreted results and edited the manuscript.

\section{ACKNOWLEDGEMENTS}

The authors would like to thank Dr. Phil G. Campbell and Dr. Lee E. Weiss of Carnegie Mellon University, PA, Dr. Prasanna Ekambaram and Dr. Peter C. Lucas of University of Pittsburgh, PA, for their experimental insights, advice and encouragement.

\section{CONFLICTS OF INTEREST}

The authors declare no conflicts of interest.

\section{FUNDING}

This work has been supported in part by NIH grants 
R01 CA168628 and R21 CA204644 to TL Whiteside, by the Deutsche Forschungsgemeinschaft to M-N Theodoraki (research fellowship \# TH 2172/1-1) and by the Dowd Fellowship from the College of Engineering at Carnegie Mellon University to S. Yerneni.

\section{REFERENCES}

1. Marur S, Forastiere AA. Head and Neck Squamous Cell Carcinoma: Update on Epidemiology, Diagnosis, and Treatment. Mayo Clin Proc. 2016; 91: 386-96.

2. Ferris RL, Blumenschein GJ, Fayette J, Guigay J, Colevas AD, Licitra L, Harrington K, Kasper S, Vokes EE, Even C, Worden F, Saba NF, Iglesias Docampo LC, et al. Nivolumab for Recurrent Squamous-Cell Carcinoma of the Head and Neck. N Engl J Med. 2016; 375: 1856-67.

3. Seiwert TY, Burtness B, Mehra R, Weiss J, Berger R, Eder JP, Heath K, McClanahan T, Lunceford J, Gause C, Cheng JD, Chow LQ. Safety and clinical activity of pembrolizumab for treatment of recurrent or metastatic squamous cell carcinoma of the head and neck (KEYNOTE-012): an open-label, multicentre, phase 1b trial. Lancet Oncol. 2016; 17: 956-65.

4. von Beckerath MP, Reizenstein JA, Berner AL, Nordqvist KW, Landstrom FJ, Lofgren AL, Moller CG. Outcome of primary treatment of early laryngeal malignancies using photodynamic therapy. Acta Otolaryngol. 2014; 134: 852-8.

5. Succo G, Rosso S, Fadda GL, Fantini M, Crosetti E. Salvage photodynamic therapy for recurrent nasopharyngeal carcinoma. Photodiagnosis Photodyn Ther. 2014; 11: 63-70.

6. Lorenz KJ, Maier H. Photodynamic therapy with metatetrahydroxyphenylchlorin (Foscan) in the management of squamous cell carcinoma of the head and neck: experience with 35 patients. Eur Arch Otorhinolaryngol. 2009; 266: 1937-44.

7. Lorenz KJ, Maier H. [Squamous cell carcinoma of the head and neck. Photodynamic therapy with Foscan]. [Article in German]. HNO. 2008; 56: 402-9.

8. Castano AP, Mroz P, Hamblin MR. Photodynamic therapy and anti-tumour immunity. Nat Rev Cancer. 2006; 6: 53545 .

9. Garg AD, Nowis D, Golab J, Agostinis P. Photodynamic therapy: illuminating the road from cell death towards antitumour immunity. Apoptosis. 2010; 15: 1050-71.

10. Mroz P, Vatansever F, Muchowicz A, Hamblin MR. Photodynamic therapy of murine mastocytoma induces specific immune responses against the cancer/testis antigen P1A. Cancer Res. 2013; 73: 6462-70.

11. Theodoraki MN, Lorenz K, Lotfi R, Furst D, Tsamadou C, Jaekle S, Mytilineos J, Brunner C, Theodorakis J, Hoffmann TK, Laban S, Schuler PJ. Influence of photodynamic therapy on peripheral immune cell populations and cytokine concentrations in head and neck cancer. Photodiagnosis Photodyn Ther. 2017; 19: 194-201.
12. Sleeman JP. The metastatic niche and stromal progression. Cancer Metastasis Rev. 2012; 31: 429-40.

13. Kalluri R, Weinberg RA. The basics of epithelialmesenchymal transition. J Clin Invest. 2009; 119: 1420-8.

14. Thiery JP. Epithelial-mesenchymal transitions in tumour progression. Nat Rev Cancer. 2002; 2: 442-54.

15. Jechlinger M, Grunert S, Beug H. Mechanisms in epithelial plasticity and metastasis: insights from $3 \mathrm{D}$ cultures and expression profiling. J Mammary Gland Biol Neoplasia. 2002; 7: 415-32.

16. Niessen K, Fu Y, Chang L, Hoodless PA, McFadden D, Karsan A. Slug is a direct Notch target required for initiation of cardiac cushion cellularization. J Cell Biol. 2008; 182: 315-25.

17. Uzdensky A, Kolpakova E, Juzeniene A, Juzenas P, Moan J. The effect of sub-lethal ALA-PDT on the cytoskeleton and adhesion of cultured human cancer cells. Biochim Biophys Acta. 2005; 1722: 43-50.

18. Casas A, Sanz-Rodriguez F, Di Venosa G, Rodriguez L, Mamone L, Blazquez A, Jaen P, Batlle A, Stockert JC, Juarranz A. Disorganisation of cytoskeleton in cells resistant to photodynamic treatment with decreased metastatic phenotype. Cancer Lett. 2008; 270: 56-65.

19. Tsai T, Ji HT, Chiang PC, Chou RH, Chang WS, Chen CT. ALA-PDT results in phenotypic changes and decreased cellular invasion in surviving cancer cells. Lasers Surg Med. 2009; 41: 305-15.

20. Vonarx V, Foultier MT, Xavier de Brito L, Anasagasti L, Morlet L, Patrice T. Photodynamic therapy decreases cancer colonic cell adhesiveness and metastatic potential. Res Exp Med (Berl). 1995; 195: 101-16.

21. Rousset N, Vonarx V, Eleouet S, Carre J, Kerninon E, Lajat $\mathrm{Y}$, Patrice T. Effects of photodynamic therapy on adhesion molecules and metastasis. J Photochem Photobiol B. 1999; 52: $65-73$.

22. Runnels JM, Chen N, Ortel B, Kato D, Hasan T. BPD-MAmediated photosensitization in vitro and in vivo: cellular adhesion and betal integrin expression in ovarian cancer cells. Br J Cancer. 1999; 80: 946-53.

23. Della Pietra E, Simonella F, Bonavida B, Xodo LE, Rapozzi V. Repeated sub-optimal photodynamic treatments with pheophorbide a induce an epithelial mesenchymal transition in prostate cancer cells via nitric oxide. Nitric Oxide. 2015; 45: 43-53.

24. Mao W, Sun Y, Zhang H, Cao L, Wang J, He P. A combined modality of carboplatin and photodynamic therapy suppresses epithelial-mesenchymal transition and matrix metalloproteinase-2 (MMP-2)/MMP-9 expression in HEp-2 human laryngeal cancer cells via ROS-mediated inhibition of MEK/ERK signalling pathway. Lasers Med Sci. 2016; 31: 1697-705.

25. Whiteside TL. The effect of tumor-derived exosomes on immune regulation and cancer immunotherapy. Future Oncol. 2017; 13: 2583-92. 
26. Ludwig S, Floros T, Theodoraki MN, Hong CS, Jackson EK, Lang S, Whiteside TL. Suppression of Lymphocyte Functions by Plasma Exosomes Correlates with Disease Activity in Patients with Head and Neck Cancer. Clin Cancer Res. 2017; 23: 4843-54.

27. Whiteside TL. Exosomes and tumor-mediated immune suppression. J Clin Invest. 2016; 126: 1216-23.

28. Franzen CA, Blackwell RH, Todorovic V, Greco KA, Foreman KE, Flanigan RC, Kuo PC, Gupta GN. Urothelial cells undergo epithelial-to-mesenchymal transition after exposure to muscle invasive bladder cancer exosomes. Oncogenesis. 2015; 4: e163.

29. Min H, Sun X, Yang X, Zhu H, Liu J, Wang Y, Chen G, Sun X. Exosomes Derived from Irradiated Esophageal Carcinoma-Infiltrating $\mathrm{T}$ Cells Promote Metastasis by Inducing the Epithelial-Mesenchymal Transition in Esophageal Cancer Cells. Pathol Oncol Res. 2018; 24: 11-8.

30. Greening DW, Gopal SK, Mathias RA, Liu L, Sheng J, Zhu HJ, Simpson RJ. Emerging roles of exosomes during epithelial-mesenchymal transition and cancer progression. Semin Cell Dev Biol. 2015; 40: 60-71.

31. Xu J, Lamouille S, Derynck R. TGF-beta-induced epithelial to mesenchymal transition. Cell Res. 2009; 19: 156-72.

32. Liu PF, Kang BH, Wu YM, Sun JH, Yen LM, Fu TY, Lin YC, Liou HH, Lin YS, Sie HC, Hsieh IC, Tseng YK, $\mathrm{Shu} \mathrm{CW}$, et al. Vimentin is a potential prognostic factor for tongue squamous cell carcinoma among five epithelialmesenchymal transition-related proteins. PLoS One. 2017; 12: e0178581.

33. Mahmood MQ, Ward C, Muller HK, Sohal SS, Walters EH. Epithelial mesenchymal transition (EMT) and non-small cell lung cancer (NSCLC): a mutual association with airway disease. Med Oncol. 2017; 34: 45.

34. Graves CA, Abboodi FF, Tomar S, Wells J, Pirisi L. The translational significance of epithelial-mesenchymal transition in head and neck cancer. Clin Transl Med. 2014; 3: 60 .

35. $\mathrm{Wu} \mathrm{Y,} \mathrm{Sarkissyan} \mathrm{M,} \mathrm{Vadgama} \mathrm{JV.} \mathrm{Epithelial-}$ Mesenchymal Transition and Breast Cancer. J Clin Med. 2016; 5 .

36. Yao X, Sun S, Zhou X, Zhang Q, Guo W, Zhang L. Clinicopathological significance of ZEB-1 and E-cadherin proteins in patients with oral cavity squamous cell carcinoma. Onco Targets Ther. 2017; 10: 781-90.

37. Angadi PV, Patil PV, Angadi V, Mane D, Shekar S, Hallikerimath S, Kale AD, Kardesai SG. Immunoexpression of Epithelial Mesenchymal Transition Proteins E-Cadherin, beta-Catenin, and N-Cadherin in Oral Squamous Cell Carcinoma. Int J Surg Pathol. 2016; 24: 696-703.

38. Sun H, Liu M, Wu X, Yang C, Zhang Y, Xu Z, Gao $\mathrm{K}$, Wang $\mathrm{F}$. Overexpression of $\mathrm{N}$-cadherin and betacatenin correlates with poor prognosis in patients with nasopharyngeal carcinoma. Oncol Lett. 2017; 13: 1725-30.

39. Thiery JP, Acloque H, Huang RY, Nieto MA. Epithelial- mesenchymal transitions in development and disease. Cell. 2009; 139: 871-90

40. Yang J, Mani SA, Donaher JL, Ramaswamy S, Itzykson RA, Come C, Savagner P, Gitelman I, Richardson A, Weinberg RA. Twist, a master regulator of morphogenesis, plays an essential role in tumor metastasis. Cell. 2004; 117 : 927-39.

41. Blanco MJ, Moreno-Bueno G, Sarrio D, Locascio A, Cano A, Palacios J, Nieto MA. Correlation of Snail expression with histological grade and lymph node status in breast carcinomas. Oncogene. 2002; 21: 3241-6.

42. Yokoyama K, Kamata N, Hayashi E, Hoteiya T, Ueda N, Fujimoto R, Nagayama M. Reverse correlation of E-cadherin and snail expression in oral squamous cell carcinoma cells in vitro. Oral Oncol. 2001; 37: 65-71.

43. Theodoraki MN, Lorenz KJ, Schneider J, Thierauf JC, Spagnoli G, Schuler PJ, Hoffmann TK, Laban S. Influence of Photodynamic Therapy on the Expression of Cancer/ Testis Antigens in Squamous Cell Carcinoma of the Head and Neck. Anticancer Res. 2016; 36: 3973-82.

44. Korbelik M, Sun J. Cancer treatment by photodynamic therapy combined with adoptive immunotherapy using genetically altered natural killer cell line. Int J Cancer. 2001; 93: 269-74.

45. Thong PS, Ong KW, Goh NS, Kho KW, Manivasager V, Bhuvaneswari R, Olivo M, Soo KC. Photodynamictherapy-activated immune response against distant untreated tumours in recurrent angiosarcoma. Lancet Oncol. 2007; 8: 950-2.

46. Hong CS, Funk S, Muller L, Boyiadzis M, Whiteside TL. Isolation of biologically active and morphologically intact exosomes from plasma of patients with cancer. J Extracell Vesicles. 2016; 5: 29289.

47. Theodoraki MN, Yerneni SS, Hoffmann TK, Gooding WE, Whiteside TL. Clinical Significance of PD-L1(+) Exosomes in Plasma of Head and Neck Cancer Patients. Clin Cancer Res. 2018; 24: 896-905.

48. Lin CJ, Grandis JR, Carey TE, Gollin SM, Whiteside TL, Koch WM, Ferris RL, Lai SY. Head and neck squamous cell carcinoma cell lines: established models and rationale for selection. Head Neck. 2007; 29: 163-88.

49. Muthuswamy R, Mueller-Berghaus J, Haberkorn U, Reinhart TA, Schadendorf D, Kalinski P. PGE(2) transiently enhances DC expression of CCR7 but inhibits the ability of DCs to produce CCL19 and attract naive T cells. Blood. 2010; 116: 1454-9. 